\title{
A Rare Case of Vertebral Osteomyelitis and Bilateral Psoas Abscess From an Unknown Source
}

\author{
Henrik Ghantarchyan ${ }^{\text {a, b, d }}$, Simi Philip ${ }^{c}$, Kathleen Dunn ${ }^{c}$, Jin S. Suh
}

\begin{abstract}
We present a case of a 31-year-old Filipino man with vertebral osteomyelitis and bilateral psoas abscesses. Vertebral osteomyelitis is a rare infection of the bone and bone marrow that affects the vertebral column. The patient's initial presentation included a 1-week history of cough, fatigue, diarrhea, fevers, and a 40-pound weight loss. An extensive workup including a computed tomography (CT) scan of the abdomen and pelvis demonstrated bilateral psoas abscesses with subcutaneous air tracking into the right flank. Blood cultures revealed $E s$ cherichia coli, Streptococcus anginosus, and Bacteroides. However, with an unclear primary source of infection, the most likely causative factor we believed to be due to immobility.
\end{abstract}

Keywords: Bilateral psoas abscess; Vertebral osteomyelitis; Sedentary lifestyle

\section{Introduction}

Vertebral osteomyelitis can arise from either bacterial direct inoculation, hematogenous spread, or contiguous spread from a soft tissue infection [1]. The initial presentation can vary from localized abdominal or flank pain or fever. One of the major complications of a vertebral osteomyelitis includes a paravertebral abscess.

The possible etiologies include intravenous drug use, human immunodeficiency virus (HIV) infection and renal failure. Trauma, hematoma formation and surgery on adjacent structures can also predispose to development of psoas abscesses [2]. Bilateral psoas abscesses are rare and can lead to significant complications, including thrombosis, hydronephrosis, hydroureter, small bowel obstruction, septic arthritis, and osteomyelitis of the vertebrae, hip, and sacroiliac joints [3].

Manuscript submitted August 11, 2020, accepted August 18, 2020

Published online September 23, 2020

aSt. Joseph's University Medical Center, 703 Main St., Paterson, NJ 07503, USA bSt. George's University, School of Medicine, Grenada, West Indies, USA 'Department of Infectious Disease, St. Joseph's University Medical Center, 703 Main St., Paterson, NJ 07503, USA

dCorresponding Author: Henrik Ghantarchyan, St. Joseph's University Medical Center, 703 Main St., Paterson, NJ 07503, USA. Email: hghantar@sgu.edu

doi: https://doi.org/10.14740/jmc3565

\section{Case Report}

A 31-year-old Filipino man with a history of cough variant asthma presented with a cough, fatigue, diarrhea, and fevers. An extensive history revealed a previous employment as a nurse in the Philippines; however, the patient admitted to a sedentary lifestyle, including approximately $18 \mathrm{~h}$ a day of playing video games in addition to a poor diet. The patient adapted this lifestyle after migrating from the Philippines to the USA for 6 months. A computed tomography (CT) scan of the abdomen and pelvis demonstrated bilateral psoas abscesses with extensive subcutaneous air tracking into the right flank. A subsequent magnetic resonance imaging (MRI) revealed early S1 osteomyelitis. Laboratory results included the following: white blood cell (WBC) 22.8 (85\% neutrophils; reference range: 3.4 - 48), absolute lymphocytes 0.64 (reference range: $\left.0.90-4.6 \times 10^{3} / \mathrm{mm}^{3}\right)$, immature granulocytes absolute $0.2 \times$ $10^{3} / \mu \mathrm{L}$ (reference range: $0.0-0.1 \times 10^{3} / \mu \mathrm{L}$ ), erythrocyte sedimentation rate (ESR) 61 (reference range: $0-15 \mathrm{~mm} / \mathrm{h}$ ), highsensitivity C-reactive protein (hsCRP) 55 (reference range: 0 - $10 \mathrm{mg} / \mathrm{L}$ ), HIV-1/2 Ag/Ab Combo negative, $\mathrm{CD}^{+}$helper 322 (26.8\%; reference range: $359-1,519 / \mu \mathrm{L})$, procalcitonin 0.09 (reference range: 0 - $2 \mathrm{ng} / \mathrm{mL}$ ), QuantiFERON-TB Gold negative, and urine toxicology negative.

The patient was initially managed in the operating room for an incision and drainage of psoas abscess, with cultures revealing Escherichia coli, Streptococcus anginosus, and Bacteroides thetaiotaomicron. Medical management included ceftriaxone, vancomycin, and metronidazole for appropriate antibacterial coverage. The underlying etiology remained unclear as no colonic microperforations were appreciated on a CT scan. Any history of intravenous drug abuse was also denied by the patient. Living situations included living with his mother and aunt, who both happened to be nurses.

The hospital course was complicated by a pulmonary embolism (PE). The patient's management included surgical debridement for the psoas abscess in conjunction with antibiotic therapy, as mentioned earlier, for antibiotic coverage. The patient was discharged with $6-8$ weeks of outpatient antibiotics.

\section{Discussion}

Vertebral osteomyelitis or disc space infection or discitis often presents with back pain, fever or neurological deficits [1]. Osteomyelitis of the spine is rare, representing only $2 \%$ to $4 \%$ 
of all cases. Vertebral osteomyelitis occurs most frequently in children aged 1 to 15 years and in adults aged 50 to 70 years [4]. The etiologies include intravenous drug use, HIV infection and renal failure [2], as seen in our case of a patient with immobility for about $18 \mathrm{~h}$ a day, spending most of the day sedentary, playing video games, possibly contributing to an immunodeficient state. Staphylococcus aureus is the most common organism causing vertebral osteomyelitis followed by Escherichia coli [1], with the latter being the common culprit in our patient.

A psoas abscess occurs probably as a result of hematogenous dissemination from an occult primary infectious focus or secondary to local trauma with intramuscular hematoma formation predisposing to abscess formation [5]. This was also seen in our patient, who had vertebral osteomyelitis with concomitant bilateral psoas abscess. As patients may initially present nonspecific symptomatology as malaise, fatigue, and subfebrile fever, they may demonstrate a more severe presentation such as abdominal-groin pain, low back pain, hip pain, difficulty in hip movements, high fever, loss of appetite, and weight loss [6].

Sepsis, which is another complication that can arise secondary to a sedentary lifestyle, may be associated with higher mortality rates when compared to the complications listed above. An association with increased serum markers of inflammation has been associated with sedentary lifestyle, including an increase in hsCRP. As exercise and physical activity are known to reduce the levels of hsCRP, it has been noted that elevated levels of hsCRP are associated with increased sepsis risk [7]. This was similarly seen in our patient as well, in which his hsCRP levels were elevated at $55 \mathrm{mg} / \mathrm{L}$. In a study of 30,183 patients conducted by Wang et al over a 9-year span, there were reported 2,553 patients who experienced a serious infection, which included 1,500 patients who experienced a community acquired sepsis. The two main factors considered were a decrease in exercise per week and an increase in time spent watching television per day. There were $10,240(33.9 \%)$ patients that reported they had no exercise during the week. There were also 7,317 (31.1\%) patients that reported they spent $>4 \mathrm{~h}$ a day watching television. It was concluded that sepsis rates were highest among those with both low exercise levels and an increase in hours per day spent watching television [7]. This helps support our conclusion of excess time spent playing video games being the cause of vertebral osteomyelitis and bilateral psoas abscess in our patient, who was noted to spend $18 \mathrm{~h}$ a day playing video games.

A proper workup can be extensive from laboratory to imaging. Modalities for infectious processes include a complete blood count, ESR, CRP, and a urinalysis. Imaging modalities of most value include ultrasound, CT scan, or MRI [6]. The treatment includes appropriate antibiotics in conjunction with surgical debridement.

In a patient with such a sedentary lifestyle with lower back pain, we recommend a consideration to include a psoas abscess. It should be taken into consideration in educating a patient into adapting a lifestyle with an increase in daily activity and avoiding sitting for long periods of time.

\section{Conclusions}

We conclude the presentation of an otherwise healthy 31-year- old Filipino man with vertebral osteomyelitis and bilateral psoas abscesses with cultures showing evidence of Escherichia coli, Streptococcus anginosus, and Bacteroides with an unknown origin of the bacteria. We consider the cause of the bilateral psoas abscess secondary to a sedentary lifestyle of playing video games for $18 \mathrm{~h}$ a day, thus contributing to an immunodeficient state.

\section{Acknowledgments}

None to declare.

\section{Financial Disclosure}

None to declare.

\section{Conflict of Interest}

None to declare.

\section{Informed Consent}

\author{
Not applicable.
}

\section{Author Contributions}

HG wrote the case report, participated in the patient care, revised and edited the case report, and approved the final version of the case report. SP participated in the patient care, revised and edited the case report, and approved the final version of the case report. KD participated in the patient care, revised and edited the case report, and approved the final version of the case report. JSS participated in the patient care, revised and edited the case report, and approved the final version of the case report.

\section{Data Availability}

The authors declare that data supporting the findings of this study are available within the article.

\section{References}

1. Giri U, Thavalathil BC, Varghese R. Vertebral osteomyelitis in an immunosuppressed patient with rheumatoid arthritis. BMJ Case Rep. 2014;2014.

2. Mannino CM, Salhab M, Schmidhofer S, Pop-Vicas A. Psoas abscess in an immunocompetent host. R I Med J (2013). 2014;97(8):32-33.

3. Ijaz M, Sakam S, Ashraf U, Marquez JG. Unusual presentation of recurrent pyogenic bilateral psoas abscess caus- 
ing bilateral pulmonary embolism by iliac vein compression. Am J Case Rep. 2015;16:606-610.

4. Tomaszewski D, Avella D. Vertebral osteomyelitis in a high school hockey player: a case report. J Athl Train. 1999;34(1):29-33.

5. Meesiri S. Septicemia of unknown origin causing by Streptococcus agalactiae primary psoas abscess: a case report. J Med Assoc Thai. 2010;93(6):735-738.
6. Askin A, Bayram KB, Demirdal US, Korkmaz MB, Gurgan $\mathrm{AD}$, Inci MF. An easily overlooked presentation of malignant psoas abscess: hip pain. Case Rep Orthop. 2015;2015:410872.

7. Wang HE, Baddley J, Griffin RL, Judd S, Howard G, Donnelly JP, Safford MM. Physical inactivity and longterm rates of community-acquired sepsis. Prev Med. 2014;65:58-64. 